\title{
ACQUISITION AND EXPANSION OF ADULT RAT BONE MARROW MULTIPOTENT MESENCHYMAL STROMAL CELLS
}

\author{
Šulla, I. ${ }^{1}$, Balik, V. ${ }^{2}$, Šarišský, M. ${ }^{3}$ \\ ${ }^{1}$ Department of Anatomy, Histology and Physiology, University of Veterinary Medicine and Pharmacy \\ Komenského 73, 04181 Košice \\ ${ }^{2}$ Department of Neurosurgery \\ ${ }^{3}$ Department of Pharmacology, P. J. Šafárik University, Faculty of Medicine, Tr. SNP 1, 04011 Košice \\ Slovakia \\ igor.sulla@uvlf.sk
}

\section{ABSTRACT}

This study was initiated in order to test a mini-invasive method of mesenchymal stem/progenitor cells (MS/PCs) isolation from a rat bone marrow (BM), and subsequently their expansion, differentiation, and evaluation of their immunophenotypic characteristics; and later their preservation as donor cells in an optimal condition for potential autotransplantation. The study group comprised of 6 adult male Sprague-Dawley (S-D) rats, weighing 480-690 g. The rats were anaesthetised by isoflurane with room air in a Plexiglas box and maintained by inhalation of a mixture of isoflurane and $\mathrm{O}_{2}$. Their femurs were surgically exposed and their diaphyses double-trephined. Then BM cells were flushed out by saline with heparin and aspirated into a syringe with a solution of DMEM (Dulbecco's modified eagle's medium) and heparin. The mononuclear cells from the $\mathrm{BM}$ were isolated by centrifugation and expanded in a standard culture medium supplemented with ES-FBS (es-cell-qualified foetal bovine serum), L-glutamine and rh LIF (recombinant human leukemia inhibitory factor). Following 14 days of passaging cultures, the cells were split into 2 equal parts. The first culture continued with the original medium. The second culture received additional supplementation with a human FGF $\beta$ (fibroblast growth factor beta) and EGF (epidermal growth factor). The populations of these cells were analysed by light-microscopy, then the mean fluorescence intensities (MFIs) of CD90 and Nestin were evaluated by a tricolour flow cytometry using monoclonal antibodies. The type of general anaesthesia used proved to be appropriate for the surgical phase of the experiments. All rats survived the harvesting of the $\mathrm{BM}$ without complications. The total number of mononuclear cells was $1.5-4.0 \times 10^{6}$ per sample and the proportion of CD90/Nestin expressing cells was $<1 \%$. Following 14 days of expansion, the cells became larger, adherent, with fibrillary morphology; the proportion of cells expressing CD90/Nestin increased to almost $25 \%$, i. e. they earned basic phenotypic characteristics of MSCs. Throughout the further cultivation a gradual decrease of the CD90/Nestin expression occurred. This suggested that the suitability of rat bone marrow derived MS/PCs for replacement therapy would probably be the highest between days $12-15$ of cultivation and then would diminish. 
Key words: adult rat; bone marrow; expansion; isolation; multipotent cells

\section{INTRODUCTION}

Bone marrow multipotent mesenchymal stromal cells (MSCs), also known as mesenchymal stem/progenitor cells (MS/PCs), represent a heterogeneous population of the non-blood producing fraction of bone marrow $[2,3,4$, 14]. Their ability to differentiate into tissues of mesenchymal origin such as bone, tendon, and cartilage or, under specific conditions, into neural, muscle, myocardial or liver cells makes them a valuable source of different types of progenitors for transplantation $[6,13,21,22]$. Being members of a team participating in a study of new approaches in the treatment of spinal cord injuries, including replacement therapy of damaged neurocytes, astrocytes and oligodendrocytes by autologous stem cells, the authors developed, and with positive results, tested a modified method of harvesting and expansion of adult rat bone marrow MSCs enabling an easy autotransplantation to donors, thus avoiding possible adverse immune reaction $[2,3,4,10,19,20]$.

\section{MATERIALS AND METHODS}

The experimental protocols were prepared with respect to the rules of the Animal Protection Act of Slovakia No. 15/1995 [1], approved by the Ethical Commission of the P. J. Šafárik University, Faculty of Medicine in Košice, as well as the State Veterinary and Food Administration of the Slovak Republic in Bratislava by the Decision No.SK P 10552/03-220. The experimental procedures in animals were performed under general anaesthesia and the postoperative painful sensations were suppressed by the intramuscular administration of anodynes. Six adult male Sprague-Dawley (S-D) rats weighing 480-690g were used in this study.

\section{Harvesting of rat bone marrow stromal cells}

The general anaesthesia was induced by inhalation of a mixture of isoflurane (1-chloro-2,2,2-trifluoroethyldifluoro-methylether) with room air in a Plexiglas box. Following the 2-3 min inhalation of anaesthetics, when the surgical phase of narcosis was achieved, animals were removed from the box and placed on a heated platform which kept their rectal temperature between 36.6 and $37.2^{\circ} \mathrm{C}$. The general anaesthesia was further maintained by administration of $1.5 \%$ isoflurane with medical oxygen $\left(\mathrm{O}_{2}\right)$ via a special mask designated for rodents. The lateral parts of both femoral regions were shaved and the exposed area of skin was disinfected by an iodine solution (Iodopolyvidonum). The disinfected skin and femoral fascia were incised, adjacent parts of musculus rectus femoris and musculus vastus lateralis were dissected and between them the ventrolateral surface of femur was approached. After removal of the periosteum, two burr-holes (one at the proximal, the second at the distal end of diaphysis ossis femoris) were performed using a tooth-drill. The bone marrow from the femoral medullary cavity was flushed by $2 \mathrm{ml}$ of saline with $0.1 \mathrm{ml}$ of heparin (solutio heparini natrici sterilisata et titrata 5000 u.i.) instilled into one of the burr-holes and aspirated by an injection needle from the other into a syringe with $2 \mathrm{ml}$ of collecting medium "Invitrogen", i.e. Dulbecco's modified eagle's medium (DMEM) with heparin under aseptic conditions (Fig. 1). After aspiration of the bone marrow $(\mathrm{BM})$, the dissected thigh muscles were fixed in their original position by several non-absorbable stitches, then the femoral fascia and skin were sutured in anatomic layers. The position of the rat was changed, the skin of the contralateral thigh was disinfected, incised, and the femur was approached, double-trephined and the bone marrow was harvested from the opposite side, also. Before finishing the experimental operation, antibiotics (amoxicillinum natricum with kalii clavulans) were administered intramuscularly in a total dose of $30 \mathrm{mg}$ and $2 \mathrm{ml}$ of anodyne tramadol [2-dimethylamino-methyl-1-1-(3-methoxyphenyl)cyclohexanol] was injected intramuscularly at two different places. After suture of the second surgical wound, the rat was transferred to a disinfected cage, where it was provided with drinking water ad libitum and food granules. Two randomly chosen rats were chosen as shame controls. Blood samples $(0.5 \mathrm{ml})$ aspirated by a thin needle from the right heart ventricle were collected into a syringe containing $0.2 \mathrm{ml}$ of heparin solution just before finishing the administration of general anaesthesia.

\section{Cultivation of multipotent stromal cells}

Immediately after aspiration of the BM from both femurs, the material in the collecting medium was removed to a special cell culture laboratory at the Department of 
Pharmacology, P. J. Šafárik University, Faculty of Medicine. The medium was dissected into small pieces, homogenized and filtered through a $70 \mu \mathrm{m}$ filter (to remove bone fragments), diluted 1:1 with Hank's balanced salt solution (HBSS) and mononuclear cells were isolated by Ficoll-Urographin density gradient centrifugation (DCG) at $1600 \mathrm{rpm}$ for 30 minutes. After centrifugation, the mononuclear cells were collected from the interface, washed two times in DMEM, suspended at $10^{6}$ cells. $\mathrm{ml}^{-1}$ in culture media and plated in 12 well culture dishes. The cells were cultured in alpha-MEM (minimum essential medium) supplemented with $10 \%$ ES-FBS (embryonic stem-cell-qualified foetal bovine serum), $2 \mathrm{mM}$ L-glutamine, $10 \mathrm{ng} \cdot \mathrm{ml}^{-1}$ human rh LIF (recombinant human leukemia inhibitory factor), 100 u.i./ $\mathrm{ml}^{-1}$ PNC (benzylpenicillinum kalicum) and $10 \mu \mathrm{g} \cdot \mathrm{ml}^{-1}$ STM (streptomycinum sulphate), half volume of the culture solution being replaced with a fresh medium every three days.

At passage 4 (after 14 days of cultivation), the cell cultures were divided into two equal parts. In the first subgroup of cultures, we continued to use the same medium, whereas in the second subgroup, the medium was additionally supplemented with rh FGF- $\beta$ (recombinant human basic fibroblast growth factor beta) and rh EGF (recombinant human epidermal growth factor). At the 14th, 21st, 36th and 45th day of cultivation (passages P4, P7, P12, P15) the samples of cells were stained with a combination of FITC/PE/PECy5, i.e. monoclonal antibodies (mouse IgG1) against rat CD45 (thyrosine phosphatase expressed by haemopoietic cells), CD90 (protein expressed by mesenchymal cells) and cy-Nestin (type VI intermediate filament-associated protein expressed mostly by nerve cells) and the mean fluorescence intensities (MFIs) were evaluated by the three-colour flow cytometry $[3,12]$.

\section{RESULTS}

The general anaesthesia by vapours of isoflurane with $\mathrm{O}_{2}$ met our expectations. Its induction phase was short (2-3 min.), the course of the narcosis was smooth, its depth sufficient for performing the surgical phase of the procedure, and subsequent awakening of the animals was uneventful and quick. All six rats survived the aspiration of the bone marrow without complications, incisions of skin, fascia, and dissections of thigh muscles; the trephinations of the femurs healed by first intention (Fig. 2).
The light-microscopic analyses of the cells isolated from the fresh adult rat bone marrow showed that a total number of mononuclear cells was 1.5-4.0 million per sample and the proportion of CD90/Nestin expressing multipotent cells was less than $1 \%$. After 14 days of in vitro expansion, the proportion of CD90 and cy-Nestin expressing cells increased to $19.2-24.9 \%$ in individual samples. The cells were large, adherent with fibrillary morphology (Fig. 3). They expressed inconsistently for CD45 (a phenotype characteristic for haemopoietic lineage), but the cells were strongly positive for CD90 and cy-Nestin, compatible with the basic phenotypic characteristics of MSCs (Fig. 4). $\mathrm{P} 2$ - primary data space region 2 . In the P2 area are located stem/progenitor cells strongly expressing CD90 marker. In flow cytometric images their colour is green. The MFIs for CD90 cells were 9552, 9356 and 9250; for nestin 3620, 2681 and 1628. Throughout the further cultivation the gradual decrease of expression of CD90, as well as cy-Nestin was observed. At passages 7, 12 and 15, i. e. after 21, 36 and 45 days of expansion, the MFIs of CD90 decreased to 9320, 8772, 6671 and of cy-Nestin to $3605,1757,541$.

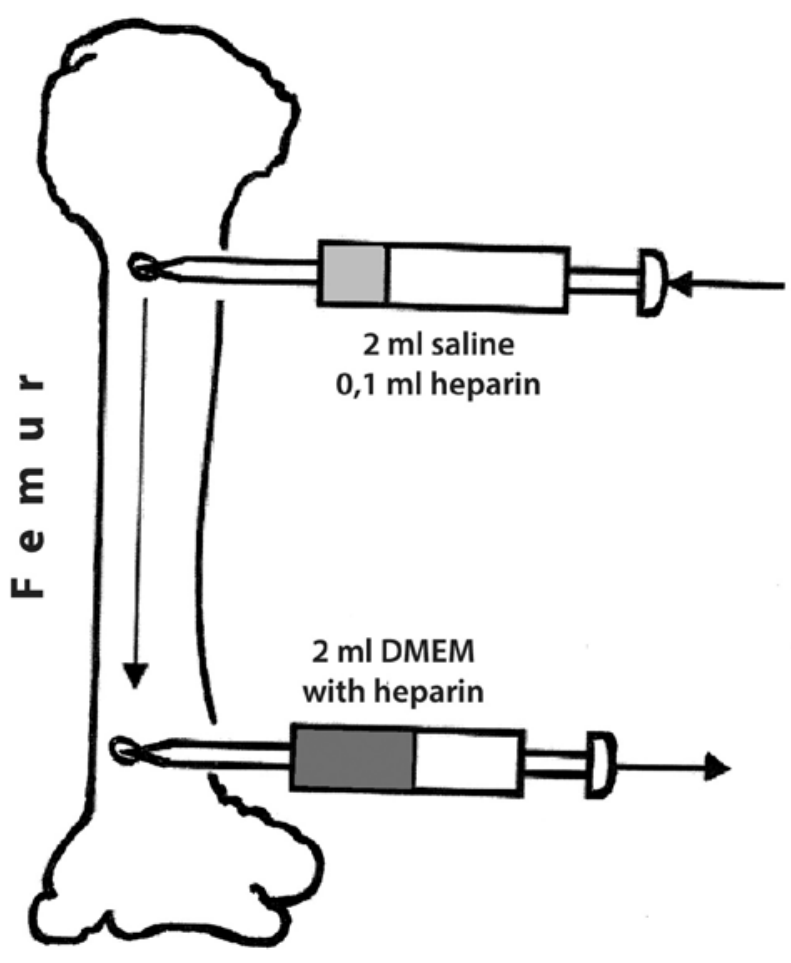

Fig. 1. Pattern of double-trephination perfusion technique of harvesting rat bone marrow cells 


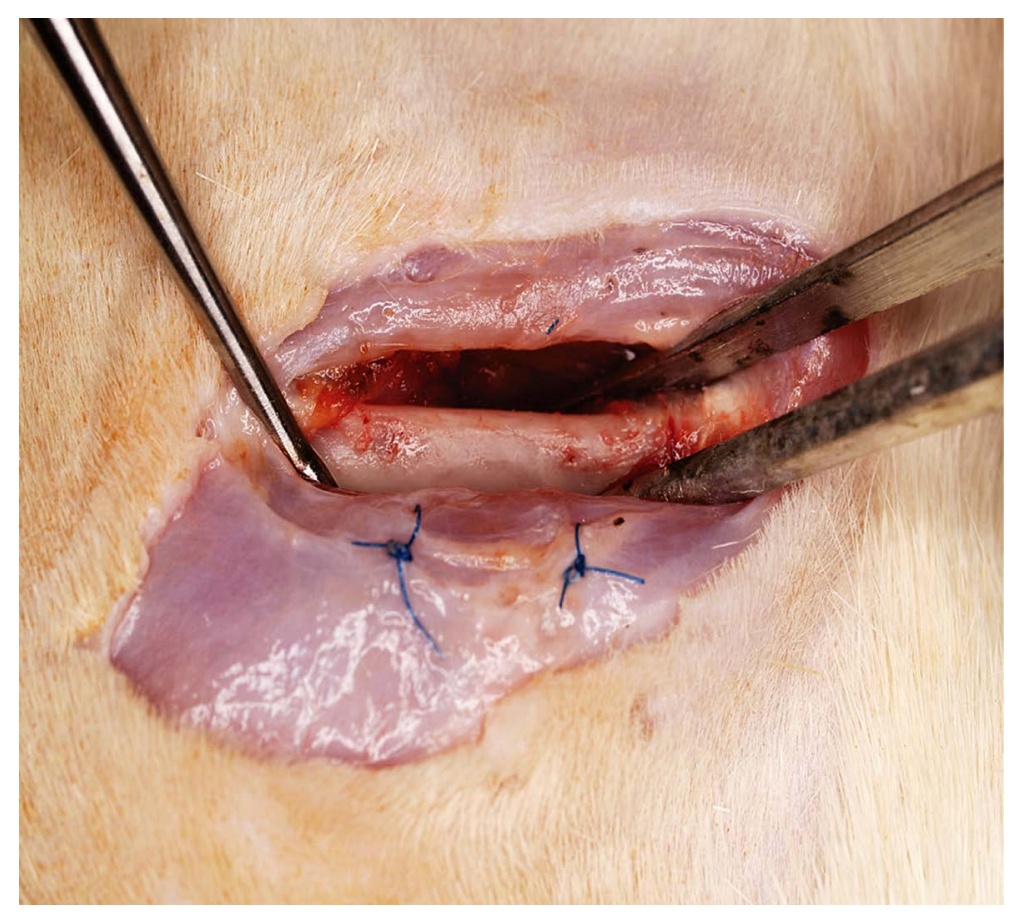

Fig. 2. Situation two months following aspiration of bone marrow. Trephinations in a rat femoral bone are almost completely healed

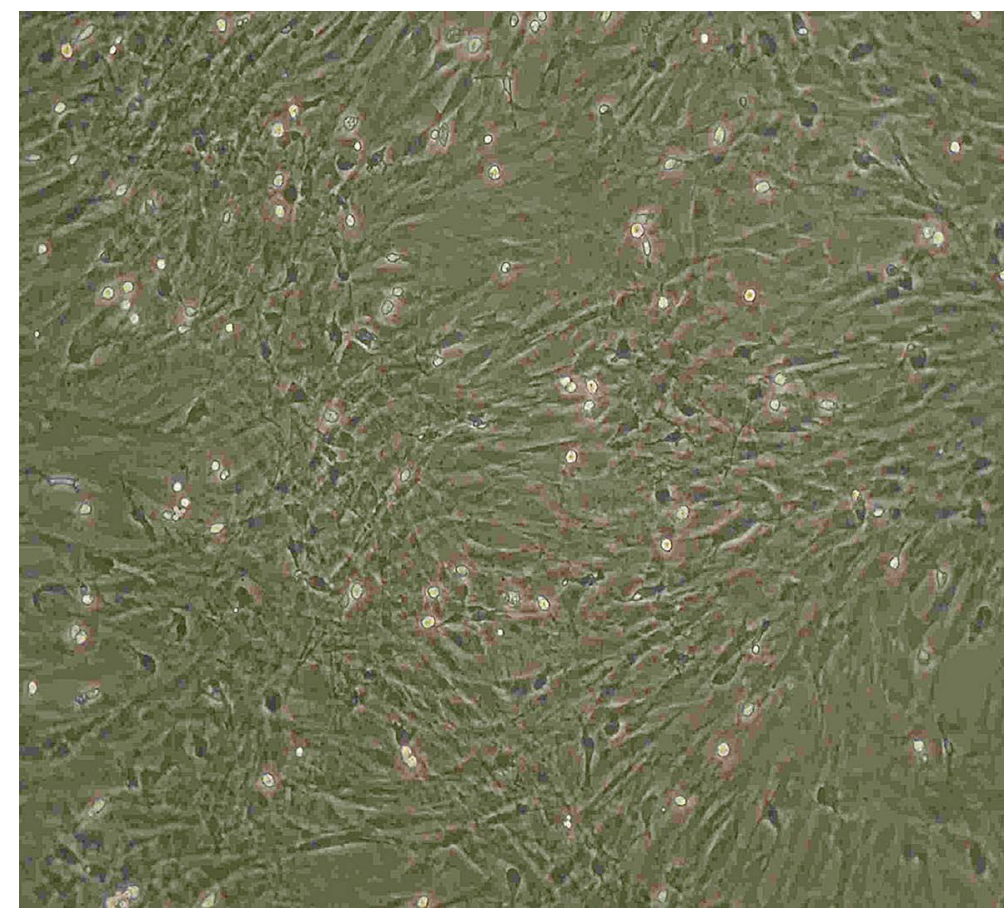

Fig. 3. A heterogeneous culture of spindle-shaped fibroblastic morphology adherent cells obtained after the initial 14 days of cultivation of bone marrow cells (passage 0 ). Original magnification Magn. $\times 100$ 

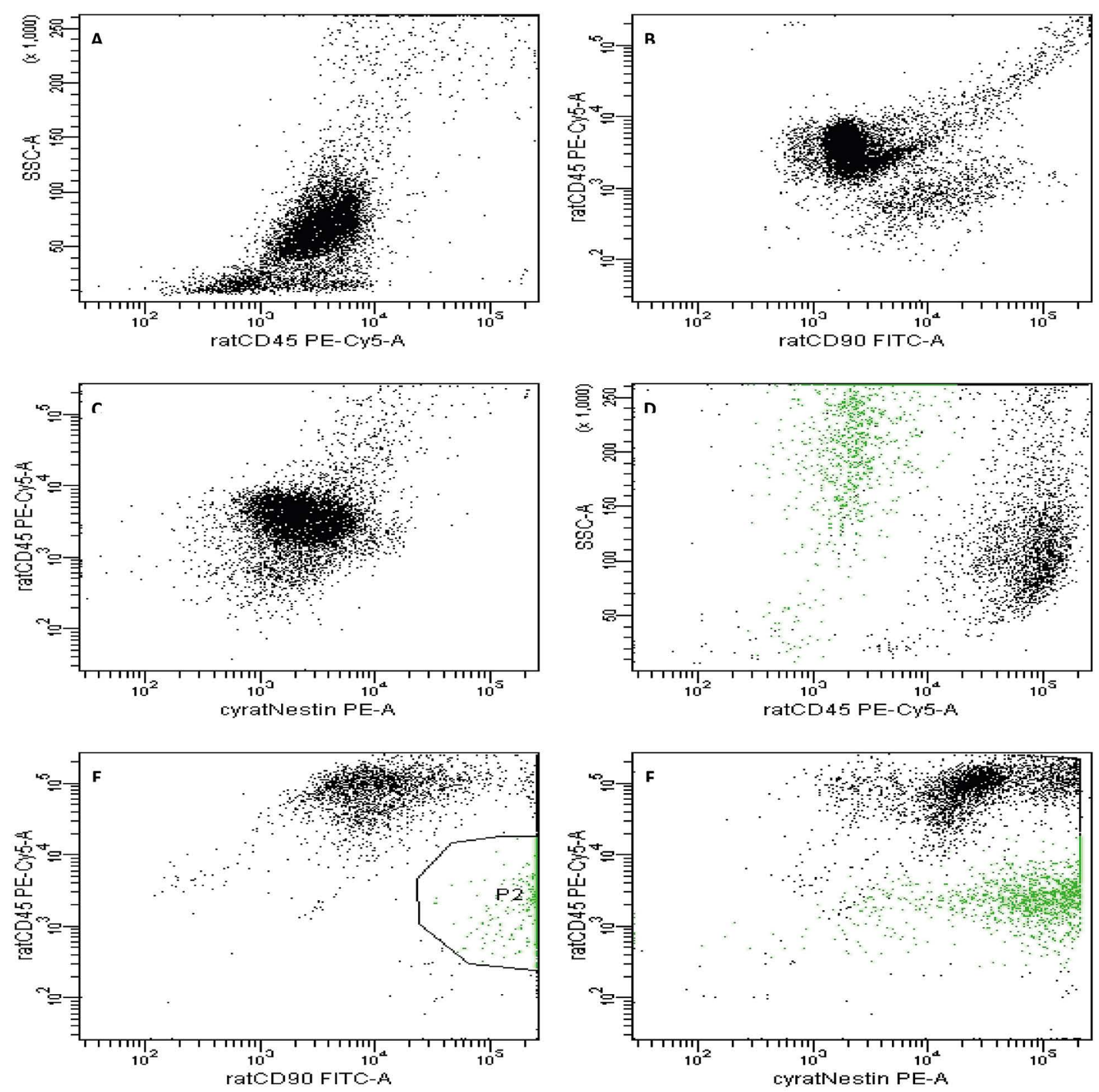

Fig. 4. Results of flow cytometry immunophenotyping analysis of rat bone marrow derived stem cells. Illustrative bivariate dot plots show expression of CD45, CD90 and nestin by the cells before (A, B, C) and after initial 14 days - passage 3 of cultivation (D, E, F). P2 - primary data space region 2

\section{DISCUSSION}

Spinal cord and brain injuries, ischemic or haemorrhagic strokes, neurodegenerative disorders (e.g. Parkinson's and Alzheimer's disease, and amyothrophic lateral sclerosis), muscular dystrophy, myocardiopathies, and myocardial infarctions, belong to serious pathological conditions connected with high morbidity and mortality [6, $10,13,21,22]$. The treatment modalities of these diseases are limited and attempts to renew impaired or lost functions are unsuccessful in the majority of cases, so far $[3,6$, 17]. The well-known fact, that the viability of tissues during the whole lifespan of an animal, is maintained by replacement of damaged cells through a process provided by special cells, called stem/precursor cells (SC/PCs), inspired scientists to use SC/PC transplants to treat different defects of organs with restricted regenerative capacity $[3,4,5,6$, $13,15]$. 
In general, there are five distinct groups of stem cells (SCs): i.e.

1. Totipotent SCs (able to differentiate into all embryonic as well as extraembryonic structures),

2. Pluripotent SCs (able to differentiate into cells of all three basal germ layers, i.e. ectoderm, mesoderm and endoderm),

3. Multipotent SCs (able to give rise to a limited SC types, e. g. skin, cartilage, bone marrow, and neurocytes),

4. Oligopotent SCs (able to differentiate only into a few SC types, such as lymphoid or myeloid SCs), and

5. Unipotent SCs, (which are able to produce only one cell type) [3]. Embryonal stem cells (ESCs) are the best source of omnipotent stem cells as they have an unlimited potential to produce any specialised cell type of an organism $[3,16]$.

Experimental results suggest the almost unrestricted capacity for their utilisation in replacement therapy and regenerative medicine $[16,17]$. On the other hand, the fact that a blastocyst developing from a fertilized egg has to be destroyed to obtain ESCs, their use is highly controversial and considered an act of violating human rights in many countries. Later on, experimental studies confirmed the possibility to acquire MS/PCs from several tissues of adult mammals. MS/PCs occur, e. g. in bone marrow, brain subventricular zone, olfactory bulb, adipose tissue, skin, and even malignant tumours $[3,4,5,11,12,13]$. They are not omnipotent or pluripotent as embryonal or foetal stem cells, however their capacity to expand, transdifferentiate into multipotent progenitors and eventually replace damaged cells, produce growth factors and trophic mediators is not substantially reduced $[13,14,15,21]$. Since the utilisation of adult MS/PCs avoids, on one hand emotional and legal problems, but on the other hand, it permits successful autotransplantation; this practice has become standard in animal experiments, as well as in clinical trials $[2,3,4,5,6$, $10,13,14,15,22]$.

There is a well established cell laboratory at the Department of Pharmacology, P. J.Šafárik University, Faculty of Medicine in Košice, specialized in the diagnostics of different types of leukemias and bone marrow diseases. This laboratory has also been able to perform phenotypic analyses of cancer cells obtained from patients after surgical resection of their tumours. The sufficient experience with the bone marrow research and high-class equipment at their disposal inspired the authors to concentrate on various problems accompanying harvesting, cultivation, expansion and transdifferentiation of multipotent mesenchymal stem cells derived from bone marrow of adult organisms. Several scientific teams dealing with SCs/PCs research have published reports recommending experimental models using different mammals and their tissues in basic spinal cord injury and transplantation research - from mice, rats and rabbits to cats, dogs, minipigs, exceptionally apes or human embryonal, foetal and adult tissues $[2,3,4,5,6,7,8,9,11$, $14,15,16,17,18,21]$. The authors decided to use S-D rats in the presented study. Their decision was influenced by several facts: namely, rats are much bigger than mice; S-D rats are more robust than Wistar rats, so they tolerate better the stress associated with complex and repeated surgical procedures; the volume of bone marrow (BM) in their femurs are greater; and the aspiration of BM from both femurs does not endanger the life or health of experimental animals.

Various techniques of bone marrow MSCs acquisition have been tested recently $[4,6,15,18]$. The majority of them require longitudinal splitting of femurs and excochleation of the cellular contents of the medullary cavity, or resection of both ends of a femur and flushing out the bone marrow from the diaphysis. In consequence, the donors are severely mutilated and perish, which sometimes is emotionally distressing and it excludes autotransplantation of cultivated MSCs. With an aim to avoid these disadvantages, the authors developed a less invasive surgical procedure. The operative technique developed proved simple and straight- forward; the amount of obtained bone marrow cells was sufficient; and the procedure did not increase the morbidity or mortality of the experimental animals. The authors believe their technique could be recommended as a method of choice for experimental acquisition of rat $\mathrm{BM}$ derived MSCs (BMDMSCs).

For the expansion of BMDMSCs, commercially available standard culture media and supplements were used which were acquired from well-established firms, such as: BD Biosciences, USA, GIBCO Products International Inc., USA, Chemicon International Inc., USA and Stemcell Technologies Inc., Canada. Their products are expensive, but of high quality, and provided reliable results. The preparation of culture media and all manipulations with cell cultures were performed in a special box with laminar air circulation at the stable temperature of $37.0^{\circ} \mathrm{C}$, in a room air containing approximately $20 \%$ of $\mathrm{O}_{2}$ (considering the 
altitude of Košice at 208 metres above the sea level). Some authors point out that hypoxic environments (oxygen concentrations 2-3\%) markedly increase the expansion of MSCs, without negative influence to the multilineage differentiation capabilities [8]. The construction of the cell culture box that the authors had at their disposal did not allow to work in such an atmosphere. In addition, it was not necessary - the amount of BM derived MSCs increased substantially during the first four passages. The acquired cells were viable and expressed essential markers characteristic for mesenchymal and neural SCs. The laboratory equipment at the Department of Pharmacology, P. J. Šafárik University, Faculty of Medicine allowed us to test only CD45, CD90 and cy-Nestin expressions. The more detailed identification of cells made ready for actual transplantation is recommended [7]. The authors plan to adopt this protocol in future transplantation experiments.

Since cy-Nestin is predominantly expressed by NS/PCs, the expression of this marker is considered to be the first step in the progression of BMDSCs along the neural lineage [19]. Following 14 days in vitro cultivation of BMDSCs in alpha-MEM solution supplemented with $10 \%$ ES-FBS, $2 \mathrm{mM}$ l-glutamine and $10 \mathrm{ng} \cdot \mathrm{ml}^{-1}$ human rh LIF, the proportion of CD45 (marker characteristic for haemopoietic lineages at all stages of leukocyte's development) was negative or \pm , but SC/PCs expressing CD90 (a cell surface marker expressed mainly by mesenchymal stem cells - MSCs) and cy-Nestin expressing cells raised from less than $1 \%$ to $19.2-24.9 \%$.

As a next step in their research, the authors decided to find out, if BMDMSC/PCs would be able to maintain their original properties, i. e. the strong $(+++)$ expression of $\mathrm{cy}-$ Nestin and CD90 markers, and none or weak $( \pm)$ expression of CD45. The gradual decrease of both, CD90 as well as cy-Nestin markers expression, confirmed by a decline of the mean fluorescence intensities of these markers during medium-term cultivation, was detected at the 21st, 36th and 45th day of passage. This observation indicates, that BMDMSC/PCs are most suitable for replacement therapy between the 12th to the 15th day of cultivation.

The results of this study are promising, however further in vitro and in vivo experiments, aimed to detect the potential of harvested NS/PCs to differentiate into mature cells of the CNS and to determine whether they gained functional properties of neurons, astrocytes or Schwann cells, eventually cells producing growth factors or trophic mediators, will be necessary. Several studies suggest that BMDMSC/ PCs have none or only limited ability to provoke an adverse immunologic reaction $[4,21]$. The authors do not share this opinion. That is why they decided to use a minimally invasive bone marrow harvesting surgical procedure offering the opportunity to proceed with MSC/PCs autotransplantation in the same experimental animals.

\section{CONCLUSIONS}

This study demonstrated that the use of the BM-harvesting method was simple and reliable. The standard culture media supplemented with ES-FBS, L-glutamine and human rh LIF can be utilized for the expansion of BMDMS/PCs. The experimental procedure did not increase morbidity or mortality. The healing of the surgical wounds was uneventful, thus easily allowing for the autotransplantation of the expanded MSCs.

\section{ACKNOWLEDGEMENT}

The presented study was supported by the Slovak Research and Development Agency under the contract APVV20-05-2005 and VEGA grant No. 1/3361/06.

\section{REFERENCES}

1. Animal Protection Act of Slovakia No. 15/1995, part 39 (In Slovak), 1250-1255.

2. Caplan, A. I., Dennis, J. E., 2006: Mesenchymal stem cells as trophic mediators. J. Cell. Biochem., 98, 1076-1084.

3. Chesier, S.H., Kalani, M.Y.S., Lim, M., Ailles, L., Huhn, S. L., Weissman, I.L., 2009: A neurosurgeon's guide to stem cells, cancer stem cells, and brain tumor stem cells. Neurosurgery, 65, 237-250.

4. Čížková, D., Rosocha, J., Vanický, I., Jergová, S., Čížek, M., 2006: Transplants of human mesenchymal stem cells improve functional recovery after spinal cord injury in the rat. Cell. Mol. Neurobiol., 26, 1167-1180.

5. Danišovič, L., Boháč, M., Zamborský, R., Oravcová, L., Provazníková, Z., Csölönyiová, M., Varga, I., 2016: Comparative analysis of mesenchymal stromal cells from different tissue sources in respect to articular cartilage tissue engineering. Gen. Physiol. Biophysics, 35, 207-214. 
6. Dezawa, M., Ishikawa, H., Itokazu, Y., Yoshihara, T., Hoshino, M., Takeda, S. et al., 2005: Bone marrow stromal cells generate muscle cells and repair muscle degeneration. Science, 309, 314-317.

7. Dominici, M., Blane, K. L., Mueller, L., Slaper-Cortenbach, I., Marini, F. C., Krause, D.S. et al., 2006: Minimal criteria for defining multipotent mesenchymal stromal cells. The International Society for Cellular Therapy position statement. Cytotherapy, 8, 315-317.

8. Greyson, W.L., Zhano, F., Brunnell, B., Ma, T., 2007: Hypoxia enhances proliferation and tissue formation of human mesenchymal stem cells. Biochem. Biophys. Res. Commun., 358, 948-953.

9. Kalanin, P., Flešárová, S., 2006: Neuron damage elicited by cardiac arrest in a dog brain. Folia Veterinaria, 50, 73-75.

10. Kim, B. G., Hwang, D.H., Lee, S.I., Kim, E. J., Kim, S.N., 2007: Stem cell-based cell therapy for spinal cord injury. Cell Transplant., 16, 355-364.

11. Maženský, D., Flešárová, S., 2016: Importance of the arterial blood supply to the rabbit and guinea pig spinal cord in experimental ischemia. In Schaller, B. (Ed.): Ischemic Stroke Updates. Tech., Croatia, 59-86.

12. Michalczyk, K., Ziman, M., 2005: Nestin structure and predicted function in cellular cytoskeletal organisation. Histol. Histopathol., 20, 665-671.

13. Phinney, D. G., Prockop, D. J., 2007: Mesenchymal stem/ multipotent stromal cells: The state of transdifferentiation and modes of tissue repair - current views. Stem Cells, 11, 2896-2902.

14. Pittinger, M.F., Mackay, A.M., Beck, S.C., Jaiswal, R.H., Douglas, R., Mosca, J.D. et al., 1999: Multilineage potential of adult human mesenchymal stem cells. Science, 284, 143-147.
15. Rider, D. A., Dombrowski, C., Sawyer, A.A., Ng, G.H.B., Leong, D., Hutmacher, D. W. et al., 2008: Autocrine fibroblast growth factor 2 increases the multipotentiality of human adipose-derived mesenchymal stem cells. Stem Cells, 26, $1598-1608$.

16. Shroff, G., Agarwal, P., Mishra, A., Sonowal, N., 2015: Human embryonic stem cells in treatment of spinal cord injury: A prospective study. J. Neurol. Res., 5, 213-220.

17. Slovinská, L., Székiová, E., Blaško, J., Devaux, S., Salzet, M., Čížková, D., 2015: Comparison of dynamic behaviour and maturation of neural multipotent cells derived from different spinal cord developmental stages: an in vitro study. Acta Neurobiol. Exp. (Wars.), 75, 107-114.

18. Soleimani, M., Nadri, S. A., 2009: A protocol for isolation and culture of mesenchymal stem cells from mouse bone marrow. Nature Protocols, 4, 102-106.

19. Šulla, I., Bačiak, L., Juránek, I., Cicholesová, T., Boldižár, M., Balik, V., Lukáčová, N., 2014: Assessment of motor recovery and MRI correlates in a porcine spinal cord injury model. Acta Vet. Brno, 83, 393-397.

20. Šulla, I., Balik, V., Petrovičová, J., Almášiová, V., Holovská, K., Oroszová, Z., 2016: A rat spinal cord injury model. Folia Veterinaria, 60, 41-46.

21. Tropel, P., Platet, N., Platel, J. C., Noël, D., Albrieux, M., Benabid, A. L., Berger, F., 2006: Functional neuronal differentiation of bone marrow-derived mesenchymal stem cells. Stem Cells, 24, 2868-2876.

22. Žilka, N., Žilková, M., Kaznerová, Z., Šarišský, M., Cigánková, V., Novák, M., 2011: Mesenchymal stem cells rescue the Alzheimer's disease cell model from cell death induced by misfolded tau. Neuroscience, 193, 330-337.

Received October 26, 2016

Accepted December 13, 2016 